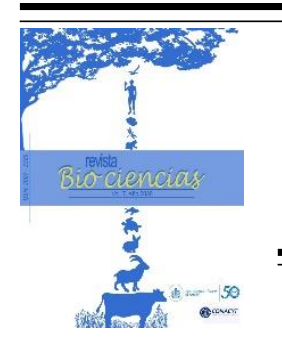

Revista Bio Ciencias

https://revistabiociencias.uan.edu.mx

ISSN 2007-3380

Memorias del 3er

Coloquio de

Nutrigenómica $y$

Biotecnología Acuícola

2020

Resumen en extenso

\title{
Afectaciones histológicas asociadas a la incidencia de Imposex en Plicopurpura pansa (Gould, 1853) después de un experimento de trasplante en tres zonas costeras de Sinaloa México.
}

\author{
González-Jiménez, J. ${ }^{*}$, Nieto-Navarro, J.T.2 , Yáñez-Rivera, B. ${ }^{3}$, Domínguez-Ojeda, $D .{ }^{2}$, \\ Betancourt-Lozano, M. ${ }^{3}$, García-Gasca, A. ${ }^{3}$. \\ ${ }^{1}$ Unidad Académica de Agricultura, Universidad Autónoma de Nayarit, Campus Xalisco Km. 9 \\ Carretera Tepic - Compostela C.P. 63780 México. \\ 2Escuela Nacional de Ingeniería Pesquera, Universidad Autónoma de Nayarit, Bahía de Matanchén \\ Km. 12, San Blas Nayarit, 63740 México. \\ ${ }^{3}$ Centro de Investigación en Alimentación y Desarrollo, A.C, Unidad Mazatlán, Av. Sábalo-Cerritos \\ s/n. Estero del Yugo, Mazatlán, Sinaloa, 82000 México. \\ *E-mail: gonzalez.jimenez02@gmail.com
}

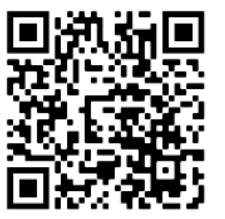

Cite this paper/Como citar este artículo: González-Jiménez, J., Nieto-Navarro, J. T., Yáñez-Rivera, B., Domínguez-Ojeda, D., Betancourt-Lozano, M., García-Gasca, A (2021). Afectaciones histológicas asociadas a la incidencia de Imposex en Plicopurpura pansa (Gould, 1853) después de un experimento de trasplante en tres zonas costeras de Sinaloa México. Revista Bio Ciencias 8: (Suppl) Memorias del 3er Coloquio de Nutrigenómica y Biotecnología Acuícola 2020 (CONYBA) e1099. http://doi.org/10.15741/revbio.08Suppl.e1099

\begin{abstract}
Resumen
Se evaluó el imposex en el gasterópodo Plicopurpura pansa en tres zonas costeras de Mazatlán Sinaloa México, enfocándose en: 1) Identificar zonas con alta y baja incidencia de imposex; 2) Realizar un experimento de trasplante con hembras morfológicamente normales a los sitios identificados con alta incidencia y 3) Determinar si las hembras trasplantadas presentaban daño gonadal a nivel histológico. Se identificaron dos sitios con alta incidencia de imposex; Cerro del Vigía (17\%) y Mármol (13\%) y un sitio con bajo imposex Las Labradas (3\%). Las hembras trasplantadas no desarrollaron imposex durante tres meses de exposición, el análisis histológico mostró alteraciones en el folículo e infiltración celular.
\end{abstract}

\begin{abstract}
Imposex was evaluated in the gastropod Plicopurpura pansa in three coastal areas of Mazatlán Sinaloa Mexico, focusing on: 1) Identifying areas with high and low incidence of imposex; 2) Carry out a transplantation experiment with morphologically normal females to the sites identified with high incidence and 3) Determine if the transplanted females had gonadal damage at
\end{abstract}

the histological level. Two sites with a high incidence of imposex; Cerro del Vigía (17\%) and Mármol (13\%) and a site with low imposex Las Labradas (3\%). The transplanted females did not develop imposex during three months of exposure, the histological analysis showed alterations in the follicle and cellular infiltration.

\section{Introducción}

El imposex es un síndrome caracterizado por el desarrollo de caracteres sexuales masculinos en hembras de gasterópodos marinos (Blaber, 1970) que ha sido asociado al compuesto biocida tributil estaño (TBT), prohibido en 2008 a nivel mundial por la Organización Marítima Internacional (IMO). En Europa el imposex es utilizado como indicador para el monitoreo en ecosistemas costeros afectados por el TBT en protocolos de la Convención para la Protección del Medio Ambiente Marino del Atlántico del Nordeste (OSPAR) y la Comisión de Helsinki (HELCOM). Actualmente se tiene registro a nivel mundial de aproximadamente 260 especies de gasterópodos marinos afectados por imposex en ecosistemas costeros expuestos a contaminación por compuestos organoestañosos (DomínguezOjeda et al., 2015). En México, se ha observado imposex en costas de Nayarit, Sinaloa y 
Campeche. Este trabajo se enfocó en el gasterópodo marino Plicopurpura pansa debido a su importancia ecológica, cultural, antecedentes en la región y por estar protegido por la Norma Oficial Mexicana NOM-059-SEMARNAT-2010 en la categoría de especie amenazada. Comprende el diagnóstico del imposex en tres zonas costeras de Mazatlán Sinaloa, un enfoque experimental en campo para evaluar el desarrollo de imposex por exposición a condiciones en sitios con alto nivel de imposex, incluyendo una comparación histológica entre hembras expuestas a sitios con alto imposex y hembras con imposex provenientes de los sitios identificados de alta incidencia de imposex.

\section{Materiales y métodos}

Área de estudio

Se realizaron muestreos de octubre de 2017 a marzo de 2018, en tres zonas costeras de Mazatlán Sinaloa, identificando los sitios con mayor y menor incidencia de imposex; calculado a través del porcentaje de hembras con imposex en cada sitio. Cerro del Vigía: zona influenciada por diversos factores antropogénicos: la zona portuaria y la planta de tratamiento de aguas residuales "el crestón" se identificó como zona de alto imposex (17\%). Mármol: zona sin actividades portuarias cercanas y alteraciones antropogénicas visibles.

Se identificó como zona de alto imposex (13\%). Las Labradas: zona arqueológica protegida sin afectación antropogénica visible. Se identificó como zona de bajo imposex (3\%) considerado como sitio control en el experimento de trasplante.

Determinación de imposex

La determinación de la incidencia de imposex se evaluó a partir de la recolección de organismos a lo largo de la línea de costa en la zona rocosa intermareal, examinando in situ a $P$. pansa (Domínguez-Ojeda et al., 2015), los caracoles se regresaron vivos a la zona intermareal después de realizar la identificación de imposex y colectar los parámetros biométricos. Para su colecta se contó con permiso SEMARNAT para colecta y estudios en laboratorio (oficio SGPA/DGVS/04783/17). La incidencia de imposex (I\%) se obtuvo calculando el porcentaje de hembras con imposex en cada sitio que presentaban un pseudo-pene, (DomínguezOjeda et al., 20015). utilizando la siguiente ecuación: $1 \%=(\#$ hembras con imposex $/ \#$ hembras totales) $\times 100$

Los datos se analizaron utilizando el programa estadístico GraphPad Prism versión 8.0. Se usó chi cuadrado ( $p=0.05$ ) para determinar diferencias estadísticas significativas entre las estaciones de muestreo y los niveles de imposex en las zonas evaluadas.

\section{Experimento de trasplante}

Se colectaron 90 hembras de $P$. pansa sin imposex del sitio de referencia (Las Labradas) se marcaron con pintura indeleble sobre la concha $y$ posteriormente se trasplantaron 30 hembras en cada sitio evaluado (Las labradas, Mármol y Cerro del Vigía) monitoreando mensualmente durante tres meses la presencia o ausencia de imposex (pseudo-pene) en las hembras marcadas.

Se realizó un análisis de varianza ANOVA y Wilcoxon para comparar los datos biométricos de los organismos de $P$. pansa colectados y determinar si existían diferencias significativas entre hembras con imposex, hembras sin imposex y hembras morfológicamente normales trasplantadas. Se utilizó una significancia de $\alpha=0.01$ para este análisis. Utilizando el programa estadístico GraphPad Prism versión 8.0.

\section{Histología}

Se compararon muestras de tejido gonadal de organismos de cada localidad: macho, hembra y hembra con imposex. El trabajo de histología se realizó en el laboratorio de histología de CIAD Mazatlán.

Los organismos se fijaron en solución Davidson y posteriormente se mantuvieron en etanol al $70 \%$. Después se realizaron cortes longitudinales de cada organismo y se procesaron histológicamente. Se obtuvieron muestras histológicas de $5 \mu \mathrm{m}$ y se tiñeron con hematoxilina- eosina-floxina, utilizando Cytoseal ${ }^{\mathrm{TM}} 60$.

Los criterios histológicos fueron en relación a las diferencias visibles entre hembras sin imposex y con imposex, determinando si existían en primera instancia evidencia de intersex en los tejidos gonadales y si existían alteraciones histológicas de algún tipo que no correspondieran a lo considerado normal en la especie.

\section{Resultados}

Las zonas costeras de Cerro del Vigía y Mármol presentaron una alta incidencia de imposex $(>12 \%)$, Las Labrada (<3\%). Todas las hembras con imposex examinadas presentaban la formación de un psudopene $<1 \mathrm{~mm}$, sin evidencias de vasos deferentes. Ninguna de las hembras trasplantadas desarrollo imposex después de 120 días de exposición a los sitios con alto índice de imposex (Cerro del Vigía y Mármol). No se encontraron diferencias significativas en tallas promedio entre hembras con imposex entre las zonas evaluadas $(P$ $<0.05$ ). El ancho y peso promedio fue mayor en hembras con imposex en comparación con las hembras morfológicamente normales trasplantadas y hembras sin imposex en las zonas evaluadas $(\alpha<0.01)$. Las tasas de recuperación de hembras marcadas trasplantadas fueron de $65 \%$ en Las labradas, $40 \%$ en Cerro del Vigía y $50 \%$ en Mármol. Las hembras trasplantadas y las hembras con imposex colectadas presentaron alteraciones histológicas severas que suponen un riesgo para la especie: folículos fragmentados, tejido conectivo irregular, infiltración celular y predominancia de ovocitos inmaduros. 


\section{Discusión}

El imposex observado en $P$. pansa no presento un vaso deferente visible similar a lo reportado en zonas costeras de Nayarit y Sinaloa (DomínguezOjeda et al., 2015). El imposex encontrado en Cerro del Vigía difiere con lo reportado en 2009 en donde se observó una incidencia del $28 \%$ (DomínguezOjeda et al., 2015). Sin embargo, esto puede deberse a que la vida promedio de una hembra de $P$. pansa es alrededor de 3.5 años en condiciones silvestres (Vázquez-Gil et al., 2004, por lo que se puede presumir que las hembras colectadas en este estudio son diferentes a las colectadas en 2009, indicando una fuente constante de imposex en la zona. De acuerdo a las clasificaciones OSPAR y HELCOM, el imposex en $P$. pansa no representaría un riesgo para la especie, sin embargo, es necesario estandarizar las metodologías a la especie de $P$. pansa dado que esta no se encuentra dentro del grupo de especies de gasterópodos modelo para evaluar el imposex. Existen evidencias en otras especies de gasterópodos donde el índice de imposex no tiene una relación directa con la concentración de TBT en el medio, tal es el caso de Marisa cornuarietis que al ser expuesta a concentraciones de 50 y 200 $\mathrm{ng} \mathrm{I}^{-1}$ de TBT durante seis meses no desarrollo más del estadio tres en la clasificación de imposex (Schulte Oehlmann et al., 1995) indicando un bajo imposex y baja contaminación de TBT en la clasificación HELCOM y no representaría un peligro para la especie. Sin embargo, estas mismas concentraciones en especies centinela como: Nucella lapillus, Nassarius reticulatus, Buccinum undatum, Neptunea antigua y Littorina littorea estarían en la clasificación seis de HELCOM la más alta y con mayor riesgo ecológico. Se encontró evidencia de alteraciones histológicas en hembras con imposex y hembras trasplantadas a sitios con alto imposex como folículos rotos, infiltración celular visible y tejido conectivo laxo deforme, coincidiendo con lo reportado en Thalessa aculeata que presento daño gonadal, encogimiento de ovocitos, apoptosis y picnosis en gónada sin embargo no hubo una correlación entre el daño histológico y la presencia de TBT en la zona (Rumampuk et al., 2018).

\section{Conclusión}

El imposex en Cerro del Vigía podría estar asociado a contaminantes antrópicos, derivados de actividades portuarias y urbanas, sin embargo en la zona de Mármol no existen evidencias de contaminación antrópica, por lo que se hipotetiza que el imposex en esa zona es producido por eventos naturales (se requieren estudios posteriores). De acuerdo a los convenios internacionales OSPAR Y HELCOM el imposex encontrado en los sitios Cerro del Vigía y Mármol no representa un riesgo para la especie.

\section{Referencias}

Blaber, S. J. (1970). The occurrence of a penis-like outgrowth behind the right tentacle in spent females of Nucella lapillus (L.). Journal of Molluscan Studies, $39(2-3): \quad 231-233$. https://doi.org/10.1093/oxfordjournals.mollus.a065097

Domínguez-Ojeda, D., Patrón-Soberano, O. A., Nieto-Navarro, J. T., Robledo-Marenco, M. J. and VelázquezFernández, J. B. (2015). Imposex in Plicopurpura pansa (Neogastropoda: Thaididae) in Nayarit and Sinaloa, Mexico. Revista mexicana de biodiversidad, 86(2): 531-534. https://doi.org/10.1016/i.rmb.2015.04.018

Vázquez-Gil, C. A., Cervantes-Hernández, P., Serrano-Guzmán, S. J., Cid-Rodríguez, R. P. and FuenteCarrasco, M. E. (2004). Análisis de la mortalidad en la población del caracol púrpura Plicopurpura pansa (Gould, 1853) en Bahías de Huatulco, Oaxaca. Ciencia y Mar, 8(24): 21-29. http://www.umar.mx/profesores/puerto angel/maria del rosario piedad cid.html

Schulte-Oehlmann, U., Bettin, C., Fioroni, P., Oehlmann, J. and Stroben, E. (1995). Marisa cornuarietis (Gastropoda, Prosobranchia): a potential TBT bioindicator for freshwater environments. Ecotoxicology, 4(6): 372-384. https://doi.org/10.1007/BF00118872

Rumampuk, N. D., Rumengan, I. F., Rompas, R. M., Undap, S. L., Boneka, F. B., Jensen, K. R. and Lasut, M. T. (2018). Tributyltin (TBT) contamination and impacts on imposex in Thalessa aculeata (Mollusca: Neogastropoda: Muricidae) in Minahasa Peninsula coastal waters, North Sulawesi, Indonesia. Aquaculture, Aquarium, Conservation \& Legislation-International Journal of the Bioflux Society (AACL Bioflux), 11(1). http://www.bioflux.com.ro/docs/2018.184-193.pdf 